\title{
Electron Avalanches in Liquid Argon Mixtures
}

\author{
J.G. Kim, S.M. Dardin, R.W. Kadel ${ }^{1}$, J.A. Kadyk, W.A. Wenzel \\ Lawrence Berkeley National Laboratory \\ Berkeley, CA 94720 USA \\ V. Peskov \\ Royal Institute of Technology \\ Stockholm, Sweden.
}

\begin{abstract}
We have observed stable avalanche gain in liquid argon when mixed with small amounts of xenon in the high electric field $(>7 \mathrm{MV} / \mathrm{cm})$ near the point of a chemically etched needle in a point-plane geometry. We identify two gain mechanisms, one pressure dependent, and the other independent of the applied pressure. We conclude that the pressure dependent signals are from avalanche gain in gas bubbles at the tip of the needle, while the pressure independent pulses are from avalanche gain in liquid. We measure the decay time spectra of photons from both types of avalanches. The decay times from the pressure dependent pulses decrease (increase) with the applied pressure (high voltage), while the decay times from the pressure independent pulses are approximately independent of pressure or high voltage. For our operating conditions, the collected charge distribution from avalanches is similar for $60 \mathrm{keV}$ or $122 \mathrm{keV}$ photon sources. With krypton additives, instead of $\mathrm{Xe}$, we measure behavior consistent with only the pressure dependent pulses. Neon and TMS were also investigated as additives, and designs for practical detectors were tested.
\end{abstract}

I. Introduction

The potential advantages of liquids over gases for particle detection have stimulated research on the noble liquids [1]. The intrinsic spatial resolution in liquids is better, both because the density of ions from charged particles is much greater, and because the diffusion of drifting electrons is less. Moreover, for experiments with small particle cross-sections (notably neutrino experiments), the density of the target detection medium is of utmost importance. In detectors using drifting electrons in liquid argon (LAr) [2], signals produced without avalanching are small and have a small ( 10:1) signal/noise ratio. The spatial resolution is at the $150 \mu \mathrm{m}$ level. However, if significant avalanche gain $(\geq 100)$ can be produced with this type of LAr detector, much better resolution should be possible. Reliable avalanche behavior has been achieved in high purity xenon [3], with a proportional response much as in gas avalanche counters. But results with more affordable pure argon were limited because avalanche production was erratic, unstable, and unreliable.
The present investigation was directed toward finding a more satisfactory mode of operation for a liquid argon avalanche detector. One motivation is the potential application to very large-scale $(\geq 10$ kton) neutrino detectors to observe short-lived particles in accelerator experiments, such as the $\tau$ lepton, which would leave a very short track. Very good spatial resolution is essential in this example, requiring a point resolution of $\sim 10 \mu \mathrm{m}$ for every $\sim 100 \mu \mathrm{m}$ of track length $(\sim 1 \mathrm{~mm})$. The avalanche detector would serve as both as a massive neutrino target and as a high-resolution detector. The use of pure liquid $\mathrm{Xe}$ in such applications would be prohibitively expensive, but the cost of Ar is much less and acceptable.

Here we report the observation of avalanches in liquid Ar/xenon and Ar/krypton mixtures, studied as functions of voltage, pressure, Xe concentration, radiation intensity, and gamma-ray energy. A sharp needle $(\sim 0.25 \mu \mathrm{m}$ radius tip) has been used as the anode to achieve the field enhancement required on the needle tip for electron avalanching. The concentration of $\mathrm{Xe}$ was varied to investigate the stability of operation and pulse amplitude and other

${ }^{1}$ Contact. W. 
characteristics; even small Xe concentrations were found to stabilize the performance.

Several measurements verified our understanding of the electrostatics of our apparatus. We investigated the dependences of the anode current and corresponding pulse height spectra on applied voltage for two sources with different gamma-ray energies and fluxes. Pulse height spectra and decay time of photon emission from $\mathrm{Xe}_{2}{ }_{2}$ eximers has been measured with respect to applied HV, source location, pressure, and Xe concentration.

For a better understanding of the energy transfer mechanism in electron avalanche processes in LAr, various concentrations of krypton (Kr) have been added, and the results compared to those obtained in similar tests using Xe.

\section{Experimental Setup}

\section{A. Physical Arrangement}

The experimental apparatus is shown schematically in Fig. 1. The detector consists of a chemically sharpened tungsten needle (anode), the tip of which was placed midway between two conducting planes: the lower (ground) plane and the needle (anode) are at zero DC potential, and the upper (drift electrode/cathode) is at negative $\mathrm{HV}$ (Fig. 2).

The detector is enclosed in a stainless steel "test vessel" that can be baked out and evacuated. The test vessel is a cylinder of about 3 liter total volume that is filled with $\sim 2.6$ liters of LAr for testing. It has several ports for: (a) a moveable $0.1 \mathrm{Ci}^{241} \mathrm{Am}$ source internal to the test system that illuminates the LAr with gamma rays of peak energy $60 \mathrm{keV}$ [4]; (b) a thin aluminum window ( $0.25 \mathrm{~mm}$ thickness) allowing use of an external ${ }^{57} \mathrm{Co}$ source $(122 \mathrm{keV})$ of $\sim 5 \mathrm{mCi}$ strength; (c) electrical leads for connections to the anode, cathode, temperature monitors, etc., (d) a vacuum ultraviolet (UV) PMT [5] to detect photons associated with the avalanche through a $\mathrm{CaF}_{2}$ lens and $\mathrm{MgF}_{2}$ window; (e) a single wire gas detector using $\mathrm{P} 10\left(\mathrm{Ar} / \mathrm{CH}_{4} 90 \% / 10 \%\right)$ or $\mathrm{CF}_{4}$ with a $\mathrm{CsI}$ photocathode also for UV photon detection. The UV PMT and CsI photodetectors operate at room temperature and pressure.

A capacitance probe measures the LAr level to a few millimeters. The temperature inside is monitored by four platinum resistors mounted at $\sim 1 \mathrm{~cm}$ intervals. The test vessel is cooled in an open bath of liquid argon, with some liquid nitrogen pre-cooling. Therefore, the temperature of the liquid in the test vessel during testing is typically $\sim 87^{\circ} \mathrm{K}$, the $\mathrm{LAr}$ boiling point at atmospheric pressure.

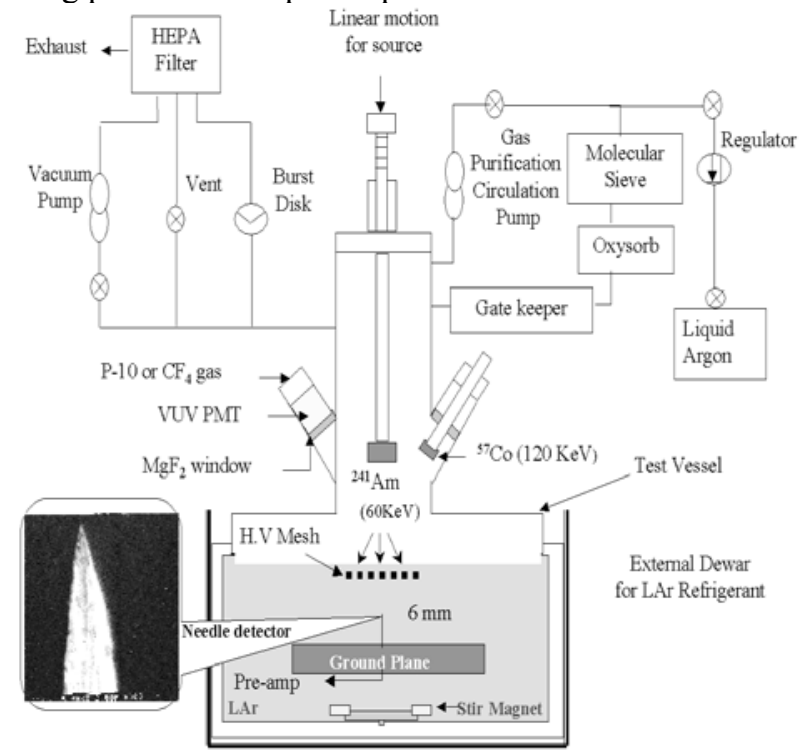

Fig. 1. A schematic of the test vessel for avalanches in liquid argon, and an electron microscope photograph of a needle tip. The $\mathrm{He}$ pressurization gas source is not indicated. Anode current and pulses are measured through electrical connections on one port

\section{B. Purity and Preparation for Tests}

To achieve the purity needed to drift electrons, the boil-off gas from the LAr supply dewar $(99.99 \%$ pure) is passed through a series of three filters: a molecular sieve [6], an "Oxysorb" $\mathrm{O}_{2}$ filter [7], and a "Gatekeeper" inert gas purifier that includes a $0.5 \mu \mathrm{m}$ particulate filter [8]. After filtering, the Ar gas to be condensed for our tests has a measured oxygen concentration $<10 \mathrm{ppb}$, the sensitivity limit of our $\mathrm{O}_{2}$ meter [9]. Tthe filter specifications [6][7][8] indicate that the $\mathrm{O}_{2}$ concentration should not exceed $1 \mathrm{ppb}$. The desired concentration of Xe is achieved by using a mass flow meter to add a calculated volume of the gas mixture $\mathrm{Ar} / \mathrm{Xe}(95 \% / 5 \%)$. We operated at gauge pressures between zero and two atmosphere $(0-30$ psig), using helium gas to maintain the pressure. The He passes through the same filters as the Ar gas. Without $\mathrm{He}$, the operating pressure of the (closed) system is approximately atmospheric pressure because of the external LAr bath.

In order to minimize impurities and outgassing, the test vessel and associated gas filling system were periodically vacuum baked at $\sim 120{ }^{\circ} \mathrm{C}$ for more than 24 hours. The base level vacuum is about $3 \times 10^{-6}$ Torr. Condensation of $\sim 2.6$ liters of LAr requires $1 \sim 2$ hours after the test vessel is cold. When the level 
LBNL-54763

indicator shows that liqufied argon has reached some intermediate level, a measured quantity of the high purity gas mixture (Ar 95\%/ Xe 5\%) is introduced directly into the test vessel to obtain the desired $\mathrm{Xe} / \mathrm{Ar}$ concentration. Chemical analysis of the input $\mathrm{Xe} / \mathrm{Ar}$ gas mixture showed the $\mathrm{O}_{2}$ concentration to be $2.6 \mathrm{ppm}$ [6]. An additional search for electronegative impurties using an ECD (Electron Capture Detector) detected none [10]. Any contamination of the $\mathrm{Xe} / \mathrm{Ar}$ gas is further diluted by a factor of $\sim 100$ when it is added to the LAr in the test vessel.

After adding the $\mathrm{Xe} / \mathrm{Ar}$ mixture, additional Ar gas is condensed to complete the 2.6 liter fill. After the filling is completed, the Ar gas supply is shut off, and the test vessel is pressurized to about $1 / 3 \mathrm{~atm}$ ( $5 \mathrm{psig}$ ) of He to suppress boiling.

A systematic change in pulse heights and rates occurs slowly during a run of several hours, which may reflect the variation of impurity concentrations in the sample with time. This effect has not been significant for the time scales of data collected here except in the case of LAr with krypton admixtures.

\section{Stirring and Sampling}

The quoted Xe concentrations assume that all the Xe dissolves uniformly in the LAr. For later tests, an alnico bar magnet mounted on a pivot near the bottom of the test vessel was rotated under the influence of an external driving magnet. This procedure mixed the liquid to insure a uniform $\mathrm{Xe} / \mathrm{Ar}$ concentration. A sampling system extracted a $\sim 10 \mathrm{ml}$ liquid sample for analysis. With heating, this liquid was expanded to the gaseous state into a oneliter sampling bottle that was shipped to an analysis laboratory [10]. Several purge-pump cycles of the liquid sampling process were repeated before obtaining a final sample.

Using this sampling technique, independent laboratory analyses were made of the $\mathrm{Xe}$ concentration dissolved in the liquid and compared the results with the concentration expected from the amount of Xe gas added to the LAr. The results are shown in Fig. 3. The expected concentration is confirmed to within about $30 \%$ for properly mixed liquids. It is clear from Fig. 3 that it is essential to have good mixing of the liquid. We believe the major systematic error in the sampling procedure is the extent to which the Xe may be concentrated in the cryogenic valve outlet during the pump/purge cycles.

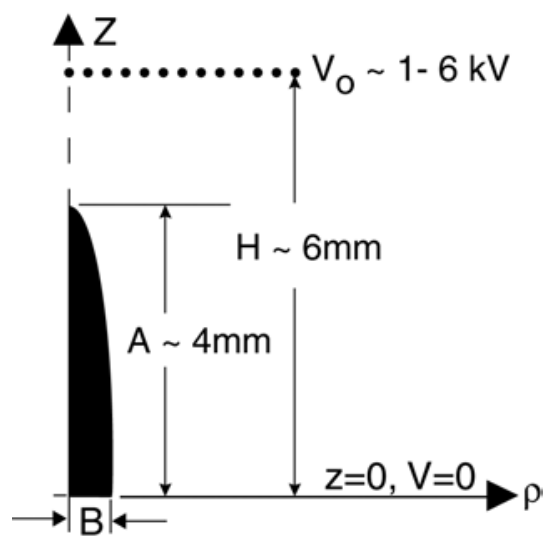

Fig. 2. The use of a prolate spheroid to simulate the needle geometry. The system is cylindrically symmetric about the $\mathrm{z}$-axis, and the screen is typically between $1-6$ $\mathrm{kV}$ with respect to the needle at ground potential. The horizontal axis is vastly expanded with respect to the vertical axis in this schematic.

\section{Prolate Spheroid Model of Needle}

As shown in Fig. 2, an electric field is produced in a gap of $\sim 6 \mathrm{~mm}$ between the wire mesh cathode and a ground plane; both electrodes are made of stainless steel. The mesh is operated, typically, at a negative potential of $1-6 \mathrm{kV}$. Protruding upward through a hole in the ground plane is an electrically isolated tungsten needle, also at dc ground potential. The needle tip is approximately halfway between the mesh and ground plane. The needle tapers from a diameter of $0.25 \mathrm{~mm}$ over most of its length, to $\sim 0.5$ $\mu \mathrm{m}$ diameter near the tip.

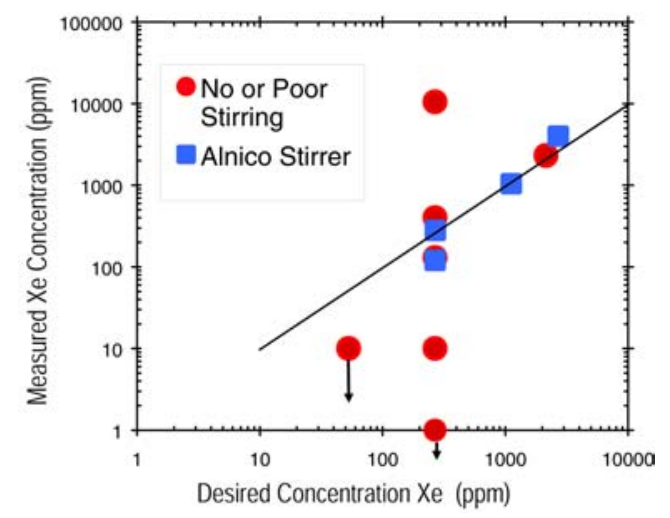

Fig. 3. Stirring and sampling results for Xe in LAr measured as a function of desired Xe concentration. The diagonal line is not a fit to the data, but indicates where the desired concentration equals the measured concentration of Xe. Downward pointing arrows (without good stirring) indicate the value is an upper limit on the Xe concentration. 
A close approximation to the actual needle geometry is that of a prolate spheroid of large aspect ratio, where $A \approx 4 \mathrm{~mm}$ is the height of the needle, the gap height $H \approx 6 \mathrm{~mm}$ and $T \approx 0.25 \mu \mathrm{m}$, is the radius of the tip (Fig. 2). $Z$ and $\rho$ are the cylindrical coordinates with $\mathrm{Z}$ along the major axis of the prolate spheroid. Smythe [11] gives the potential $V(Z, \rho)$ for the upper half prolate spheroid extending above a ground plane in a uniform electric field $E_{O}$ :

$$
V=E_{O} Z\left[1-\left(\operatorname{coth}^{-1} \eta-\frac{1}{\eta}\right) /\left(\operatorname{coth}^{-1} \eta_{O}-\frac{1}{\eta_{O}}\right)\right],
$$

where the parameters $C$ and $\eta_{o}$ and the new coordinates $(\eta, \zeta)$ and are defined by:

$$
\begin{aligned}
& C^{2}=A^{2}-B^{2}, \eta_{O}=A / C \\
& \rho=C \sqrt{\left(\eta^{2}-1\right)\left(1-\zeta^{2}\right)} \text { and } Z=C \eta \zeta .
\end{aligned}
$$

In this notation, the needle is a surface of constant $\eta$ $=\eta_{0}$ and the tip of the needle is at $\zeta=\zeta_{0}=1$. The electric field $(E(z), z=Z / C$ ) along the $Z$ axis (i.e. $\rho=0$ ) is:

$$
\frac{E(z)}{E_{o}}=1+\frac{z\left(z^{2}-1\right)^{-1}-\operatorname{coth}^{-1} z}{\operatorname{coth}^{-1} \eta_{o}-\eta_{o}^{-1}}
$$

For the large aspect ratio of the needle considered here, these expressions can be simplified to yield the electric field at the tip of the needle as:

$$
\mathrm{E}\left(\eta_{\mathrm{o}}, \zeta_{\mathrm{o}}\right)=V A /(k T H)
$$

where $k=\ln (\sqrt{A / B})-1 \sim 4.4$, for the geometry of Fig. 2. The electric field on the mesh directly above the needle is given by , $\mathrm{E}(\mathrm{mesh})=\mathrm{E}_{0}[1+2(0.03)]=$ $1.06 \mathrm{E}_{0}$, where the factor 2 comes from the image charge of the needle in the mesh, and 0.03 is the value at the mesh of the quotient on the right-handside of eqn. 3. For the dimensions of Fig. 2 the field at the tip is enhanced by a factor of 2670 over the ambient field between the mesh and the ground plane.

\section{E. Electronics and Data Acquisition}

The sensitive region between the needle and mesh is illuminated by gamma rays from either (or both) a $5 \mathrm{mCi}{ }^{57} \mathrm{Co}$ source $(122 \mathrm{keV})$ or a $0.1 \mathrm{Ci}^{241} \mathrm{Am}$ source $(60 \mathrm{keV}) ; \alpha$ particles from the latter do not penetrate a $1.2 \mathrm{~mm}$-thick steel window enclosing the source. The ${ }^{241} \mathrm{Am}$ source is moveable between 5 $\mathrm{cm}$ and $15 \mathrm{~cm}$ above the $50 \%$ optically transparent $\mathrm{HV}$ mesh. The ${ }^{57} \mathrm{Co}$ source is at a fixed position about $7.5 \mathrm{~cm}$ from the needle.

Currents from the mesh, needle, ground plane, and source holder are monitored using picoammeters [12] and recorded via the LabView program [13]. Pulses from the needle are observed on an oscilloscope. For early tests, the pulse heights and avalanche rates were monitored and stored using a pulse height analyzer (PHA) [14]. The signal from the needle is amplified first with a charge sensitive preamplifier [15], and then with an LBNL pulse-shaping amplifier ("TranLamp"), adjusted to have $5 \mu$ sec differentiation and integration time constants for pulse height measurements. The total gain (preamp and TranLamp) was about $11 \mathrm{~V} / \mathrm{pC}$ for earlier tests, and was increased by $\sim 10$ for later tests. Decay time spectra are obtained by adjusting the integration/differentiation time constants to shorter values, typically $0.1 \mu \mathrm{s} / 0.2 \mu \mathrm{s}$, respectively. The resultant current pulses from needle avalanches provided the start signal, and the PMT pulses from detected photons, delayed by $5.0 \mu \mathrm{sec}$, provided the stop signals. In later tests the PHA was replaced by a CAMAC based DAQ system with ADC and TDC information available on a pulse-by-pulse basis.

\section{Measurements using Xenon in LAr}

\section{A. Needle Current}

Electron-ion pairs are created by ionizing radiation from the ${ }^{241} \mathrm{Am}$ source in the liquid argon. The positive ions, with very low mobility, $\sim 7-11 \times 10^{-3}$ $\mathrm{cm}^{2} / \mathrm{V}$-s [16], are collected on the cathode mesh. The electrons have a much larger mobility and velocity, $\sim 3$ to $5 \mathrm{~mm} / \mu \mathrm{s}$, and are drawn to either the needle or the ground plane. The electric field at the narrow needle tip is a factor of nearly three thousand times the ambient field in the gap. Below the avalanche threshold, small signals are observed on the scope just above the noise level: these are from electrons produced in the primary ionization events, i.e., without avalanche multiplication. Above a threshold field, electron avalanching occurs. This is observable both as an increase in needle current and by a large increase in pulse height.

Based upon the known mesh voltage, and our best knowledge of the gap size, needle position and tip radius, we have calculated the electric field at the needle tip corresponding to the measured avalanche 
LBNL-54763

threshold, both analytically as described above and using a simulation program [17]. These methods are in reasonable agreement in estimating a threshold value for avalanches of about $7 \mathrm{MV} / \mathrm{cm}$ (Table I). This result is somewhat dependent on the $\mathrm{Xe}$ concentration, and is presently limited in accuracy by the uncertainty in the needle-mesh gap and the needle tip radius.

Table I. Fields at tip of needle and on the mesh for the geometry of Fig. 2 with $1500 \mathrm{~V}$ on the mesh.

\begin{tabular}{|l|l|l|}
\hline & Field at needle & Field at mesh \\
\hline $\begin{array}{l}\text { Numeric } \\
\text { Calculation } \\
\text { (Maxwell) }\end{array}$ & $7.5 \mathrm{MV} / \mathrm{cm}$ & $2.55 \mathrm{kV} / \mathrm{cm}$ \\
\hline $\begin{array}{l}\text { Analytic } \\
\text { Calculation }\end{array}$ & $6.9 \mathrm{MV} / \mathrm{cm}$ & $2.57 \mathrm{kV} / \mathrm{cm}$ \\
\hline
\end{tabular}

B. Measurement of Current and Voltage

In a test using pure LAr we measured needle current over a wide range of voltage with the ${ }^{241} \mathrm{Am}$ source. A similar test in LAr doped with $\sim 25 \mathrm{ppm}$ $\mathrm{Xe}$ a different needle and geometry, used both radioactive sources, ${ }^{241} \mathrm{Am}$ and $\mathrm{Co}^{57}$. The results are shown in Fig. 4. The data for the Xe/Ar mixture $2004 / 02 / 10 \quad 14.1$

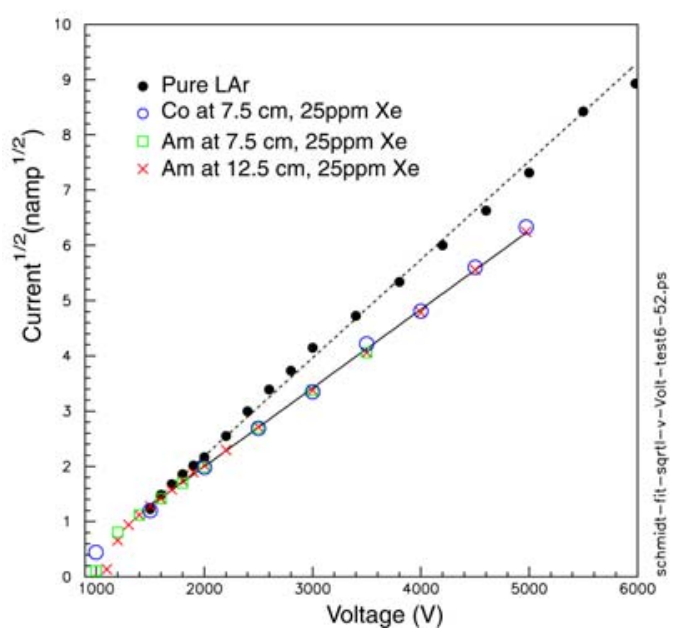

Fig. 4. Current measured versus voltage for pure LAr and LAr with $25 \mathrm{ppm}$ Xe using a different needle, and therefore different geometry. The solid curve is a fit using eqn. 5 to the LAr data with Xe $25 \mathrm{ppm}$ added, and the Am source at $12.5 \mathrm{~cm}$ (red crosses) and the dotted curve is a fit to the solid points in pure liquid argon. include three sets of measurements: 1) a ${ }^{241} \mathrm{Am}$ source ( $60 \mathrm{keV}$ photons) at $\sim 12.5 \mathrm{~cm}$ from the tip of the needle; 2) the ${ }^{241} \mathrm{Am}$ source at $\sim 7.5 \mathrm{~cm}$ from the needle; and 3) a ${ }^{57} \mathrm{Co}$ source (122 keV photons) at $\sim 7.5 \mathrm{~cm}$ from the needle. At lower voltages, the needle current induced by the ${ }^{57} \mathrm{Co}$ source is about a factor of 10 larger than for the ${ }^{241} \mathrm{Am}$ source when both are $7.5 \mathrm{~cm}$ away from the needle. This is roughly consistent with our expectations, based upon the source strengths, the difference in geometries, the difference in photon energies and corresponding absorption lengths. The measured rates of pulses from the two sources are also explained by these differences.

Because the mobility of the positive charge carriers is very small a significant space charge may build up around the needle tip. In the case of space charge limited drift, the current-voltage relationship has been calculated for the case of field emission from a needle [18] to be

$$
\mathrm{V}=\mathrm{V}_{\mathrm{vac}}+2\left(8 \mathrm{~d} / 3 \alpha \varepsilon \mu^{+}\right)^{1 / 2} \mathrm{i}^{1 / 2}(\mathrm{mks})
$$

Here, $\mathrm{d}$ is the distance from the tip of the needle to the screen $(2 \mathrm{~mm}$ or $3 \mathrm{~mm}), \varepsilon=1.51 \varepsilon_{0}$ is the dielectric constant of $\operatorname{LAr}\left(\varepsilon_{0}=\right.$ vacuum permittivity), $\mu^{+}$is the positive charge carrier mobility, $\alpha$ is a constant equal to 0.6 in the point/sphere geometry approximation [18]. $V_{v a c}$, is the voltage in vacuo necessary to produce the emitted current, and is determined by fitting the above expression to our data. This equation describes field emission from a cathode needle (hence current in vacuo), but it is expected to apply for our tests as well [16]: in both instances the current-voltage dependence is dominated by space charge limitations.

For currents greater than about $1 \mathrm{nA}$ (see Fig. 4), the $\mathrm{LAr} / \mathrm{Xe}$ data lie on a common line (solid line). Our data with pure Ar (dashed line) lie consistently above these points. We attribute the difference to the smaller distance $(\sim 2 \mathrm{~mm})$ between the needle tip and the drift electrode compared with the LAr-Xe data $(\sim 3 \mathrm{~mm})$. These data suggest that the current at higher fields is independent of the source intensity and photon energy, and is, therefore, space charge limited.

If we use $d=3 \mathrm{~mm}$ for our LAr/Xe data, we find the positive charge carrier mobility, $\mu^{+}=6.4 \times 10^{-3}$ $\mathrm{cm}^{2} / \mathrm{Vsec}$, in reasonable agreement with the value obtained by fitting our pure LAr data : $7.0 \times 10^{-3}$ $\mathrm{cm}^{2} / \mathrm{Vsec}$. These measurements are in approximate 
agreement with newer published values of the mobility of positive charge carriers in pure LAr (9.4 x $10^{-3} \mathrm{~cm}^{2} /$ Vsec [16]) but disagree by an order of magnitude with older published values $\left(\sim 6 \times 10^{-4}\right.$ $\left.\mathrm{cm}^{2} / \mathrm{Vsec}[19]\right)$. The authors of [20] conclude that for very pure LXe, the positive charge carriers are principally holes and not positive ions, because the large positive carrier mobility in LXe is inconsistent with the measured viscosity of the liquid and the hard-core radius of the Xe ions. This is also the case for Ar ions, as we have verified by an equivalent calculation of the viscosity of Ar ions in LAr. Our results lead us to a similar conclusion: the mobility for pure $\mathrm{LAr}$ with small admixtures of $\mathrm{Xe}$ is consistent only with holes, and not with $\mathrm{Xe}$ or $\mathrm{Ar}$ ions, as the primary positive charge carriers.

\section{Two Avalanche Modes}

As is evident in Fig. 5, there are for some conditions two quite distinct pulse amplitudes, whose heights do not change with source distance. Figure 5a is a picture of a oscilloscope traces clearly showing two different pulse amplitudes, also seen in the $\mathrm{PH}$ distribution of Fig 5b. As will be discussed in III-F. (Fig. 7b), the heights of the larger pulses decrease with increasing pressure. Pressure dependence of signal amplitudes was also seen by Derenzo, et al. [21]. The smaller pulses, however, are independent of the pressure. The pressure dependence of the larger pulses suggests that at least some portion of the avalanche mechanism involves the vapor phase (a "bubble" at the needle tip). However, we have no direct confirmation of this conjecture. Likewise, it is suggestive that the pressure-independent pulses are from avalanches produced entirely in liquid. In what follows, we will use the nomenclature "small pulses" and "large pulses" to refer to these two types of behavior.

\section{Variation of Pulse Height with Gap Distance}

We have tried to verify our analytic calculation of the field, and the threshold voltage for initiating avalanches in LAr/Xe by varying the geometry of our detector (see Fig. 2 and Sec. III-B.)

In Fig. 6 we show the relative pulse height versus voltage for two different values of $\mathrm{H}$, with all other dimensions held approximately constant. For the smaller gap, $\mathrm{H}=4.8 \mathrm{~mm}$, the avalanches are initiated at a voltage of $\mathrm{V} \approx 1550 \mathrm{~V}$, whereas for the larger gap of $7.7 \mathrm{~mm}$ the avalanche region starts at $\mathrm{V} \approx$ $2050 \mathrm{~V}$. The three sets of data for $\mathrm{H}=7.7 \mathrm{~mm}$ differ somewhat because of systematic effects described in section II-B. The ratio of these threshold voltages $(\sim 0.75)$, is consistent within the estimated error with that of the gap heights, $4.8 / 7.7=0.62$ (eqn. 4). This agreement provides some confidence in our description of the electric field.
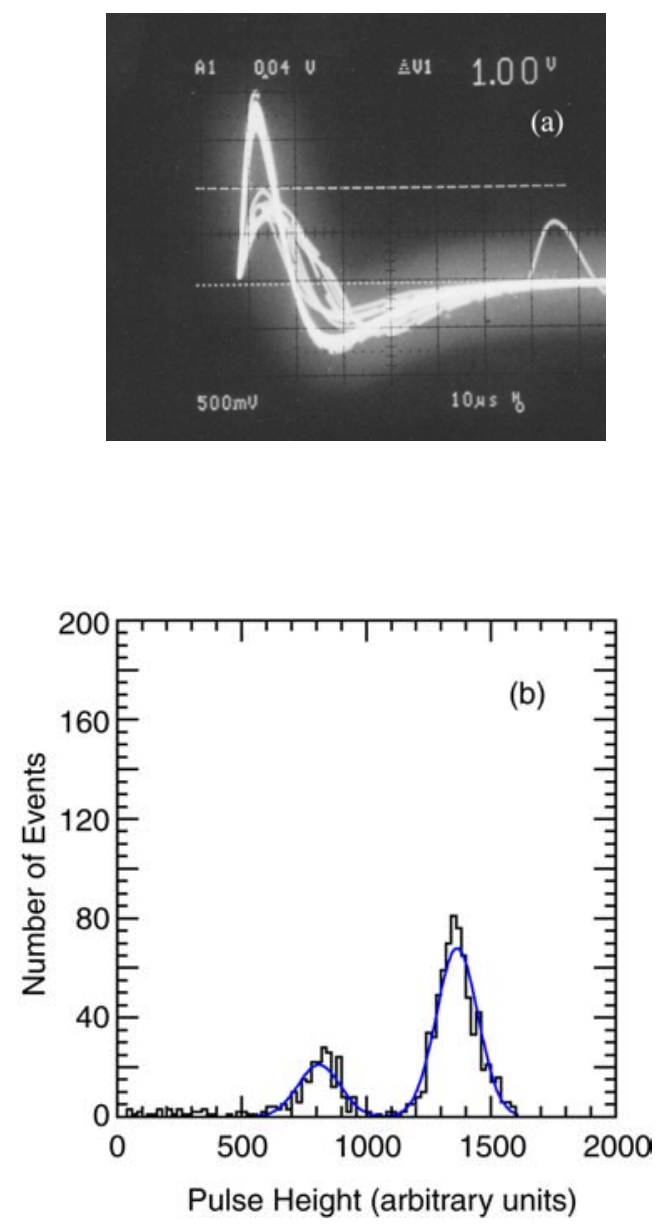

Fig. 5. (a) Oscilloscope traces of several avalanche events showing two distinct pulse heights. (50 ppm Xe, pressure 5 psig, $\mathrm{HV}=1750 \mathrm{~V}$, one large division of $\mathrm{X}$-axis is $10 \mu \mathrm{s}$, one large division of $\mathrm{Y}$-axis is $500 \mathrm{mV}$ ); (b) Pulse height distribution for the signals shown in (a). 


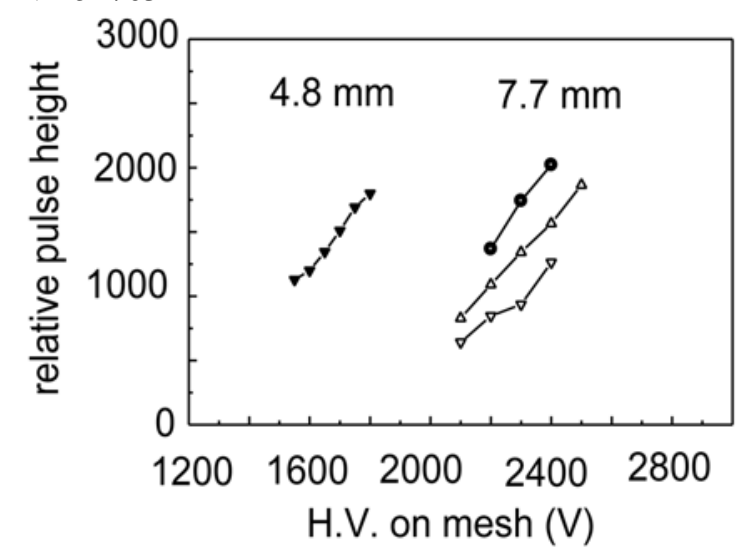

Fig. 6. Mean pulse heights of pressure dependent pulses at 5 psig $\mathrm{He}$ overpressure measured versus high voltage, for gaps of $4.8 \mathrm{~mm}$ and $7.7 \mathrm{~mm}$ (dimension $\mathrm{H}$ of Fig. 2). Results from three tests with the $7.7 \mathrm{~mm}$ gap are shown (see text). The Xe concentration for all tests is 250 ppm.

E. Pulse Height versus Voltage, Pressure and Source Position

Figs. $7 \mathrm{a}$ and $7 \mathrm{~b}$ show the dependence on voltage and pressure of the corresponding pulses shown in Fig. 5: the large pressure-sensitive pulses, and the smaller, pressure-independent pulses. Fig. 7c shows that the pulse height of both types of pulses are independent of the source position while the total rate of both pulses agrees well with a $1 /$ distance) $^{2}$ dependence. Generally, both types of pulses are uniform in shape, and have narrow pulse height distributions, as shown in Fig. 5. The large and small pulses are well within the linear region of the preamplifier and post-amplifier.

\section{F. Avalanche Gain}

We have observed avalanche pulse heights between $\sim 100 \mathrm{mV}$ and $\sim 3 \mathrm{~V}$ after amplification. These depend upon voltage, pressure and $\mathrm{Xe}$ concentration. The background noise level is approximately 10-20 $\mathrm{mV}$ peak-to-peak after amplification. Assuming that the $60 \mathrm{keV}$ gamma conversions release 2600 electrons in the liquid argon ( $23 \mathrm{eV} /$ ion pair), and using the known gain of the amplifier system, the estimated avalanche gain for the smaller pulses in Fig. 5 is $\sim 100$, assuming no ion recombination, and full contribution of all primary electrons. However, we expect recombination to be a significant $(\sim 20 \%)$ at these fields [22]. We also expect that only a fraction of the initial ionization electrons contribute to the avalanche, because the active volume is very small $(\rho=13 \mu \mathrm{m})$.
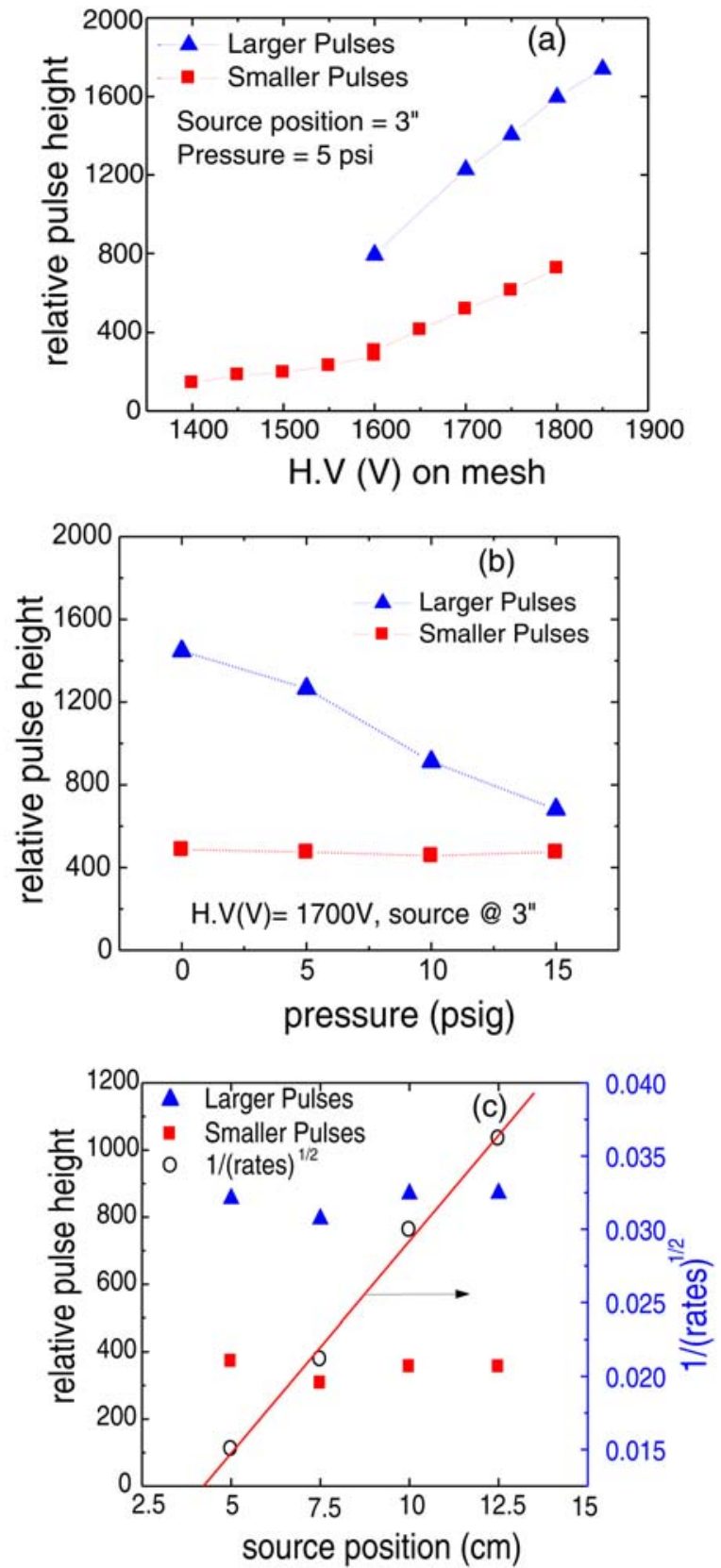

Fig. 7. (a) Pulse height vs. H.V for larger and smaller pulses. (b) Pulse height vs. pressure of He for larger and smaller pulses. (c) Pulse height and $1 /(\text { rate })^{1 / 2}$ vs. source position. The rate is the sum of larger and smaller pulses $(\mathrm{HV}=1600 \mathrm{~V}$, pressure $=5 \mathrm{psig}, \mathrm{Xe}$ concentration $=100$ ppm) 
For these reasons the actual avalanche gain is expected to be larger than $\sim 100$, but the present experimental technique does not allow a measurement of this.

\section{G. Pulse Height vs. Photon Energy}

We have measured the pulse height spectra for the ${ }^{241} \mathrm{Am}$ source at $7.5 \mathrm{~cm}$ from the needle, and the spectrum obtained when both the ${ }^{241} \mathrm{Am}$ source and the ${ }^{57} \mathrm{Co}$ source are present, each $7.5 \mathrm{~cm}$ from the needle. The resulting spectra are shown in Fig. 8.

The rate of pulses induced by the ${ }^{57} \mathrm{Co}$ source plus the ${ }^{241} \mathrm{Am}$ source is approximately five times larger than with the ${ }^{241} \mathrm{Am}$ source alone, consistent with the known source intensities and absorptive materials in front of the sources. Except for this difference in rate, the pulse height spectra are essentially identical, confirming our speculation that the avalanches are saturated, i.e. independent of the incident gamma-ray energy. This saturation may be due to the intense field created by the large avalanche charge density, i.e. a saturated avalanche in a very small volume.

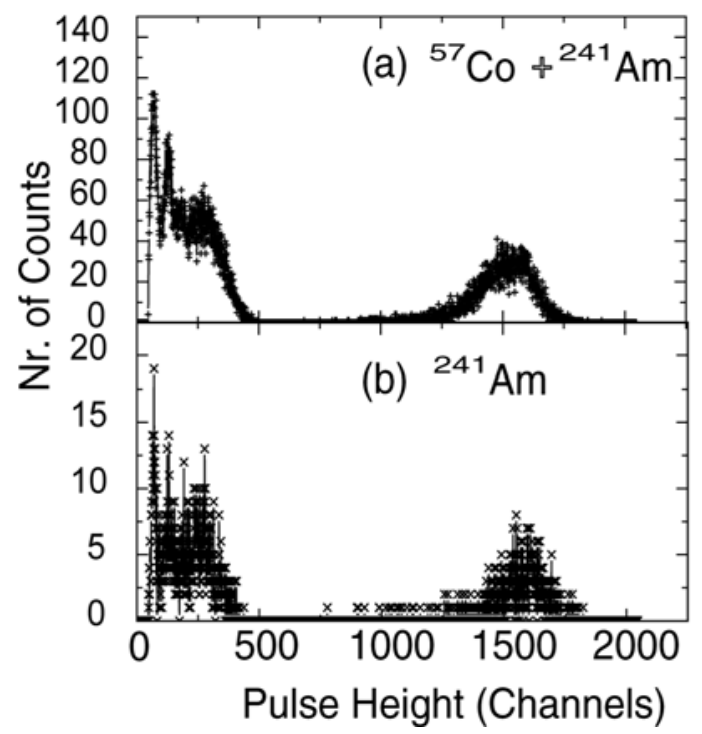

Fig. 8. Pulse height spectrum at $2200 \mathrm{~V}$ in LAr with $250 \mathrm{ppm}$ Xe, 5 psig He overpressure. The location of the peaks near channel 1500 is pressure dependent. In (a) both the ${ }^{241} \mathrm{Am}$ and ${ }^{57} \mathrm{Co}$ sources are present, and (b) is for the ${ }^{241} \mathrm{Am}$ source alone. The collection times were $240 \mathrm{sec}$ for both (a) and (b) with negligible dead-time.

\section{H. Gain vs. Xenon Concentration}

We have explored the effects of $\mathrm{Xe}$ for eight $\mathrm{Xe} / \mathrm{Ar}$ concentrations in the range of 25 to $50,000 \mathrm{ppm}$. In pure Argon, the behavior is unstable, with the pulse amplitude and rate changing over tens of seconds, or disappearing altogether, only to re-appear a few seconds later. Similar behavior is reported in [21]. Avalanche behavior is stabilized with the addition of even small amounts of Xe. Fig. 9 shows the PH of the smaller pulses versus Xe concentration at $1500 \mathrm{~V}$. One interesting behavior is that the $\mathrm{PH}$ decreases as the concentrations increase from $25 \mathrm{ppm}$ to $500 \mathrm{ppm}$, then increases at larger concentrations; it is still increasing at the greatest concentration, 50,000 ppm. An increase in ionization density for LAr with Xe concentrations above $500 \mathrm{ppm}$ was reported in [22], but it was always less than $15 \%$, and not in avalanche mode.

The pressure-dependent (larger) pulses were observed for small xenon concentrations (50 ppm to $500 \mathrm{ppm})$, but did not appear at large concentrations (1000 ppm to $50,000 \mathrm{ppm})$. In several tests, we observed a difference in performance as a function of time: typically, the PH decreased somewhat as the rate increased (or vice versa) over a period of several hours. This behavior, which occurred primarily for low concentrations, is not yet understood, but might originate from small impurities or in homogeneities in the Xe concentration. For this reason it is difficult

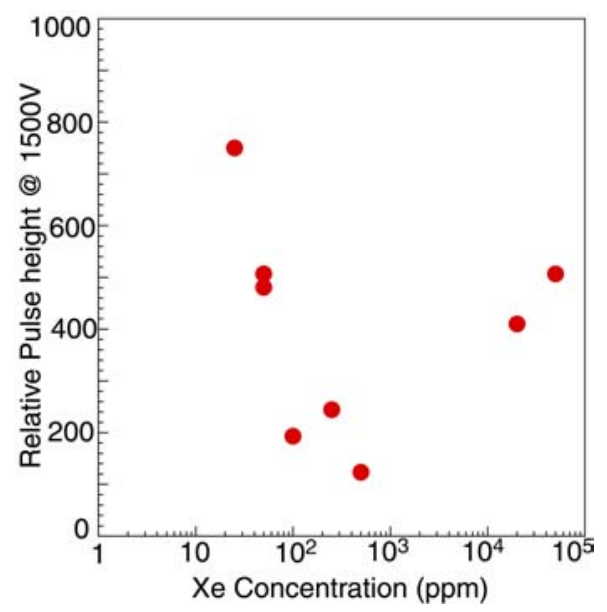

Fig. 9. Relative pulse height of smaller, pressure independent, pulses as a function of xenon concentration at $1500 \mathrm{~V}$ on the mesh, corresponding to electric fields of $\sim 9 \times 10^{6} \mathrm{~V} / \mathrm{cm}$, all taken at $\sim 5 \mathrm{psig}$. 
LBNL-54763

to quantify systematic errors on some of the measured quantities. We judge the consistency of the data based on its reproducibility from run to run.

\section{Decay Time Spectra vs. Xenon Concentration}

Processes which are known to occur in liquid $\mathrm{Xe} / \mathrm{Ar}$ mixtures include the transfer of energy from ionized Ar to Xe because of the higher ionization potential for LAr, 14.2 eV [23] compared with 10.6 $\mathrm{eV}$ for Xe dissolved in LAr [22]. Other processes are the formation, and the subsequent de-excitation of $\mathrm{Ar}^{*}{ }_{2}$ or $\mathrm{Xe}^{*}{ }_{2}$ excimers. These processes lead to emission of scintillation light in the far UV region, peaking at $128 \mathrm{~nm}$ from LAr, and $175 \mathrm{~nm}$ from LXe.

Time spectra were taken by using the needle avalanche as the start pulse on a time-to-pulse-height converter, and the detection of a UV photon in the PMT with a CsTe photocathode [5] (or a gaseous detector, see below) as the stop pulse. The decay time is determined by the de-excitation of the $\mathrm{Xe} / \mathrm{Ar}$ system. An example of such a spectrum is shown in Fig. 10. Separate tests indicate the system is capable of measuring decay constants as short as $\sim 15 \mathrm{nsec}$.

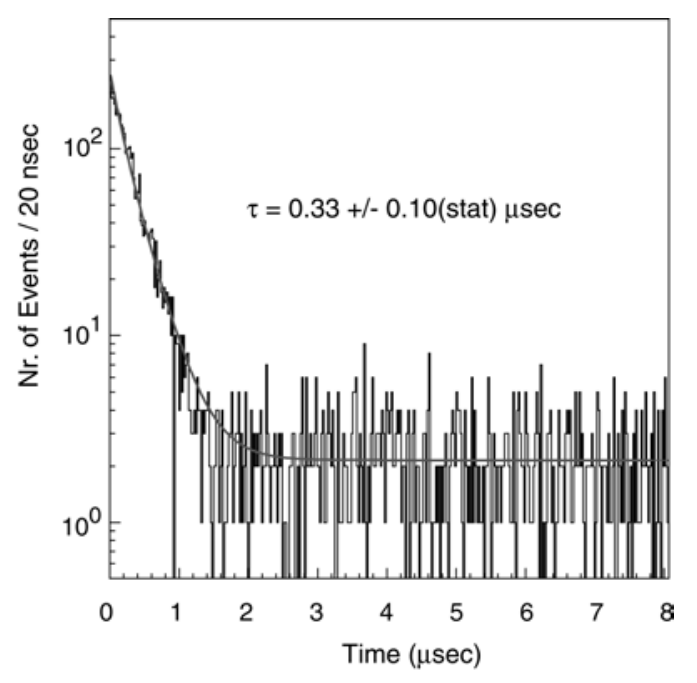

Fig. 10. Example of a time distribution for coincidences between large pulses on the needle and the PMT pulses measured with our older DAQ system. The data are fit (smooth curve) as described in the text, with the indicated decay constant. The $\mathrm{Xe} / \mathrm{Ar}$ concentration is $2000 \mathrm{ppm}, 5$ psig.

Shown in Fig. 11 is a comparison of time decay constants measured in our experiment for large pulses with those of Kubota, et al., [24] and Conti, et al., [25]. For the data shown in Figs 10 and 11, the average number of photons $(\vec{n})$ observed per event ranged between $0<\bar{n}<1.5$, depending on the test conditions. To extract the lifetime, $\tau$, we fit the decay time distribution with the function:

$$
F(t)=B \exp \left(-\bar{n}\left(1-e^{-t / \tau}\right)\right) e^{-t / \tau}+k
$$

where $B$ is a constant, $\bar{n}$ is the measured, average number of photons/event, and the constant $k$ describes the flat background. The first exponential factor represents the probability that a photon does not appear before time $t$, and the last exponential factor is the probability density for a photon appearing at time $t$. It is evident that our results are more consistent with those of Conti, et al. This result also appears to be consistent with the fact that both that experiment and ours are sensitive only to the 175 $\mathrm{nm}$ wavelength of light from Xe, but insensitive to Ar light. We have no explanation for the anomalously low time constant for our point at $50 \mathrm{ppm} \mathrm{Xe}$.

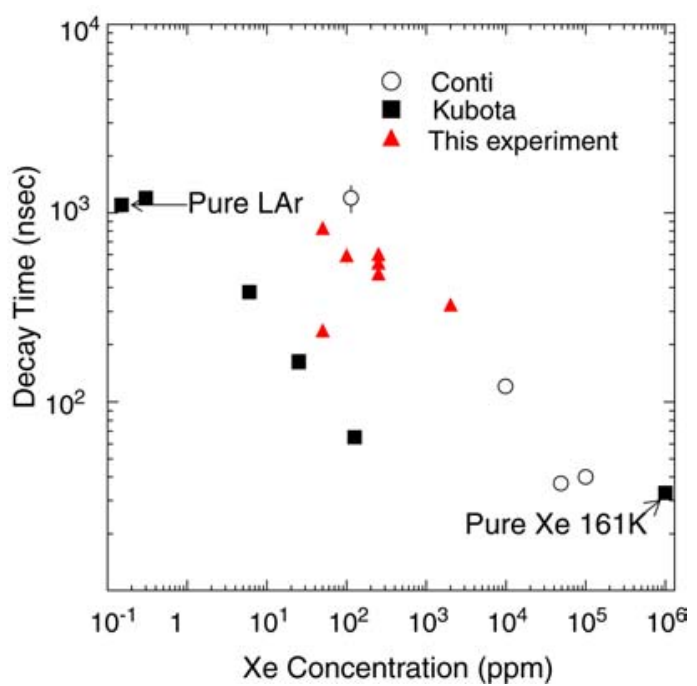

Fig 11. A comparison of our decay time constants measured as a function of Xe concentration with those of Kubota et al.[24], and Conti et. al.[25]. For our data the decay constants all correspond to large pulses at 5 psig.

Since measuring the data shown in Fig. 11 we have upgraded our DAQ system to allow simultaneous measurement of the pulse height and decay time distribution on an event-by-event basis. Fig. 12 shows typical data from this system, for 250 ppm Xe in LAr at 15 psig. We can clearly identify two different pulses, one peaking near 130 ADC counts and the second near 320 ADC counts. In the 
ADC versus TDC plot the two sets of pulses are clearly separated, and we observe that the larger pulses have a longer decay time. The apparent "curvature" of the TDC distribution of the smaller pulses when plotted versus the ADC value comes from time slewing with pulse size of the fixed threshold discriminator. We have corrected the data for time slewing by measuring the time delay of test pulses versus ADC value. The TDC distributions after correction are shown on the right hand side of the figure, along with the fit to eqn. 6 . The mean number of photons per event ( $\bar{n}$ in eqn. 6$)$ is fixed in the fit, determined independently for the large and small pulse types from the ratio of events with valid TDC data to the total number of events of each type. In this example the decay constant for the small pulses is $122 \pm 12 \mathrm{nsec}$ (stat) and the time constant for the larger pulses, $291 \pm 10 \mathrm{nsec}$ (stat).

We have investigated the systematic error on the fit, varying by $150 \mathrm{nsec}$ the common time-zero values subtracted from each event. In this exercise the fit value changes by about $6 \%$. Additionally, in cases where the photon fraction is low $(\bar{n}<<1)$, we have fit the full TDC distribution with exponential decay folded with a Gaussian resolution function plus a constant background:

$A \frac{e^{-t / \tau}}{\sqrt{2} \lambda} e^{\left(t_{0} / \tau-\sigma^{2} / 2 \tau^{2}\right)}\left(1+\operatorname{erf}\left(\left(t-t_{0}-\sigma^{2} / \tau\right) / \sqrt{2} \sigma\right)\right)+\mathrm{k}$,

where $\operatorname{erf}(x)$ is the error function:

$$
\operatorname{erf}(x)=\frac{2}{\sqrt{\pi}} \int_{0}^{x} e^{-y^{2}} d y,
$$

and the five numbers $A\left(\right.$ events/bin), $\tau(n s e c), t_{0}(n s e c)$, $\sigma$ (nsec), and $k$ (events/bin) are fit to the data. $A$ is an arbitrary amplitude, $\tau$ the decay time, $t_{0}$ is the event start, $\sigma$ is the rms of the Gaussian resolution function, and $k$ accounts for a constant background. Note, unlike eqn. 6, this fit does not take into account multiple photons, but the photon fraction for the small pulses in this case is 0.17 and results in a negligible correction according to Monte Carlo (MC) studies.

Fig. 13 shows the fit of equation (7) to the TDC data of the small pulses of Fig. 12. The two fitting procedures agree well. We estimate from these and similar studies described earlier that the fractional systematic error in the decay constant from the fitting procedure is $6 \%$ of the decay time.

We have also measured the pulse heights and decay constants for large pulses with $2.5 \mathrm{ppm} \mathrm{O}_{2}$ added to the liquid to examine the impact of electronegative contamination. With the addition of $\mathrm{O}_{2}$ the signals initially disappeared, and we had to raise the HV from $1300 \mathrm{~V}$ to $1800 \mathrm{~V}$ to recover the large pulses. The rate of small pulses was reduced to near zero. Comparisons of results for the large pulses with and without $\mathrm{O}_{2}$ are shown in Fig. 14, for the pulse height and time decay constant versus pressure.

In Fig. 14, we note that with the addition of $\mathrm{O}_{2}$ the relative pulse height only increases by only a factor of $\sim 2$ for an increase in $\mathrm{HV}$ of $500 \mathrm{~V}$, much less than expected from Fig. 7a. A possible explanation is that the $\mathrm{O}_{2}$ reduces the total charge collected, but has less impact on the decay time of the avalanche.
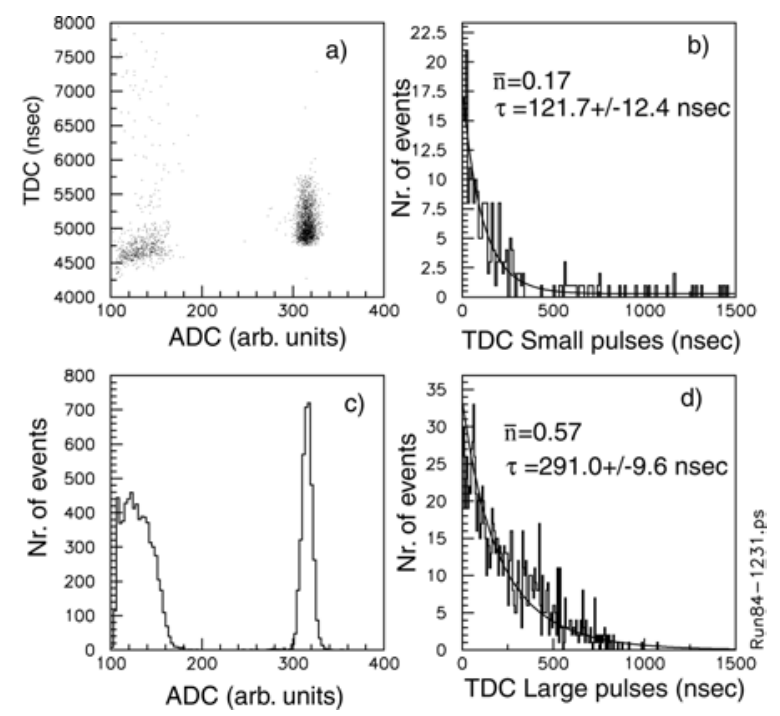

Fig. 12. ADC and TDC distributions measured for 250 ppm Xe in LAr measured at $15 \mathrm{psig}, 1300 \mathrm{~V}$. a) TDC measured versus ADC, showing both large and small pulses. Panel b) shows the TDC distributions for the small and d) large pulses fit with an exponential decay modified to account for multiple photons per event (eqn. 6). The quoted errors are statistical only. The average number of photons per event for each type of pulse is indicated, and measured as the ratio of events with non-zero TDC values divided by the total number of ADC triggers for each type. c) Pulse height distributions for the large and small pulses. 


\section{LBNL-54763}

The decay times appear similar, and we incorporate the data with $\mathrm{O}_{2}$ into the Fig. 15 which shows the variation of the decay constant as a function of pressure and high voltage. Figure 15 shows the measured decay constant for large pulses in $250 \mathrm{ppm} \mathrm{Xe}$ as a function of pressure and $\mathrm{HV}$. Three trends are apparent: 1) for the data at 5 psig the decay time has no apparent HV dependence; 2) for the higher pressure data the decay

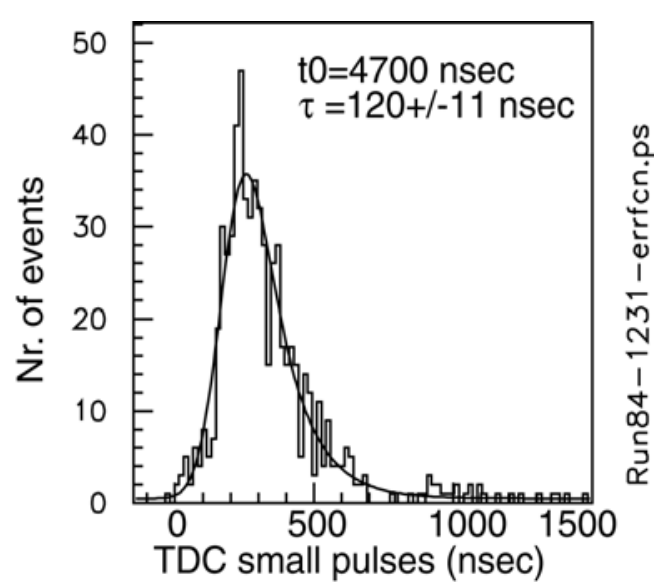

Fig. 13. Data for the small pulses of Fig. 12, fit with equation (7). There is no correction for multiple photons, and the average number of photons per event is 0.17 . The fit decay time is $120 \pm 11 \mathrm{nsec}$ (stat). Note the close agreement to the fit from eqn. 6 in Fig 12.

time decreases with increasing pressure; and 3) the decay time increases with increasing HV.

Along with the data from these runs, we have plotted in Fig. 15 the three data point at $250 \mathrm{ppm} \mathrm{Xe,}$ 5 psig of Fig 11 taken with the older DAQ. We see the data with the older DAQ system is consistent with the new DAQ system at the same pressure. We cannot fully account for the scatter of the points at 5 psig. It could reflect the pressure variations, which we can measure to about 1 psig. We note that the decay constant for the 5 psig data does not have any significant $\mathrm{HV}$ dependence (or it is masked by pressure fluctuations). Another possible explanation is that the bubble size does not vary significantly with $\mathrm{HV}$ at pressures near 5 psig (i.e. the bubble becomes "saturated" and does not exceed some finite size).
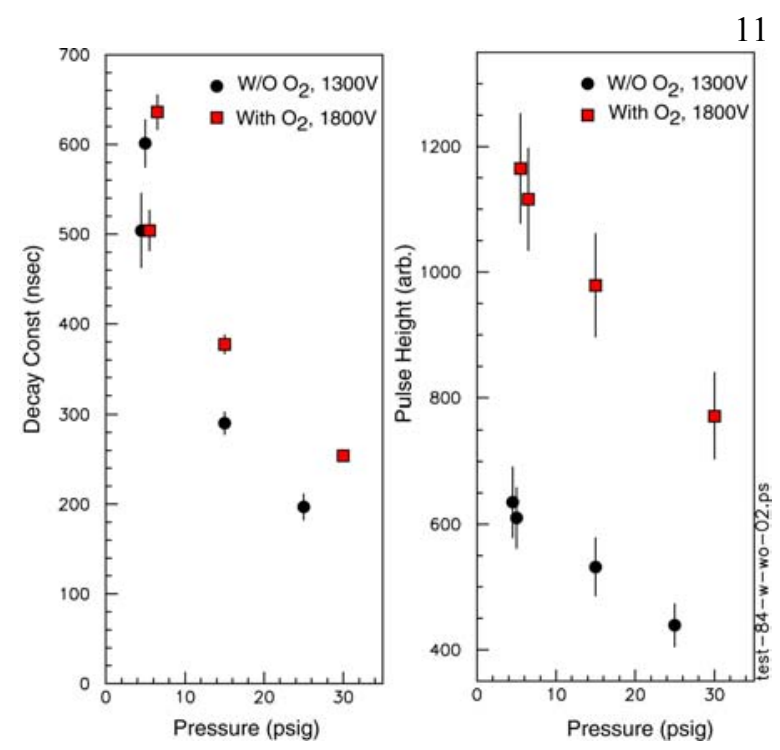

Fig. 14. Decay constants and pulse height for large pulses measured as a function of pressure for $250 \mathrm{ppm} \mathrm{Xe}$ in liquid argon (black circles) at $1300 \mathrm{~V}$, and $250 \mathrm{ppm} \mathrm{Xe}$ plus $2.5 \mathrm{ppm} \mathrm{O}_{2}$ (red squares) at $1800 \mathrm{~V}$. The points at 5 psig have been slightly displaced horizontally for clarity. The errors on the decay times are statistical only. The error bars on the pulse height data represent the rms of the ADC distributions.

At 15 and $30 \mathrm{psig}$, the decrease in the decay constant as the pressure increases seems consistent with the behavior we expect for gas bubbles. The collision time between atoms should decrease as the pressure increases, and we would expect this to shorten the apparent decay time. Alternately, the decay times measured in our experiment may be indicative of the distribution of bubble lifetimes which, in turn, depend on the HV and pressure. It is not inconsistent that the decay time increases with the $\mathrm{HV}$, because greater HV implies more energy is deposited in the bubble, which becomes larger, lengthening the decay time.

The decay times measured in our experiment are not completely reconciled with those of previous experiments [24][25]. It is relevant that the decay times measured in our experiment are the convolution of the avalanche, bubble formation, recombination and emission times, which distinguish our results from previous measurements.

Our data is consistent with [25] for the decay times versus Xe concentration, but there is no evidence of bubble formation in the experimental apparatus of [25], where the decay constant of the Xe-LAr mixture was measured using cosmic rays in a stainless steel vessel with a Suprasil fused silica window for the PMT, no electrodes, and presumably zero electric 
field. When viewed through the Suprasil window the PMT was sensitive only to Xe light, as in the present experiment. How bubbles would be made in this [25] context is difficult to explain, unless the liquid is near boiling. The authors did not quote the applied pressure or vacuum in the paper, but the pressure was expected to be approximately atmospheric pressure [26].

Our decay constants for the large pulses are much longer than those quoted in [24]. While [24] does not discuss the system pressure, previous publications by the same group indicate pressures from 2 to 3 atmospheres [27].

In Fig. 16 we plot the decay times measured for the small pulses, taken during the same run as Fig. 15. For these points we have used eqn. 7 to fit the data, in order to incorporate the most information. With one exception, the average number of photons per event is $\sim 0.1-0.2$, needing no corrections for multiple photons. We have corrected the decay constant of the remaining point at $15 \mathrm{psig}, 1000 \mathrm{~V}$ using a simple MC study, where we vary the average number of photons per event, pick the earliest photon, fit the resulting TDC distribution with eqn. 7, and determine a correction to recover the original decay time. The correction is roughly linear in the average number of photons per event. The number of photons/event in this case was 0.65 , and the fit value of $87 \mathrm{nsec}$ is corrected to $97 \pm 9 \pm 9$ (stat)(sys) nsec.

We see no evidence for $\mathrm{HV}$ or pressure dependence of these data, indicating that a different avalanche mechanism is at work. As we have previously suggested we believe the small pulses correspond to avalanches in liquid, while the large pulses correspond to avalanches in gas (bubbles).

\section{Measurements Using Krypton in Liquid Argon}

\section{A. Krypton vs. Xenon Avalanche Behavior}

As noted in section III-H, the addition of even a small amount of $\mathrm{Xe}(\leq 100 \mathrm{ppm})$ to LAr stabilized the avalanche performance. In Table II we show the cross sections and rate constants for the dissociation of Ar excimers in gaseous systems [28]. As can be seen, $\mathrm{Xe}$ is as effective in dissociating Ar dimers as a traditional additive in proportional chambers such as $\mathrm{CO}_{2}$ or $\mathrm{CH}_{4}$. The energy transfer from LAr to rare gas solutes has been shown to be dominated by collisions with Ar excitons in the lowest lying $\mathrm{n}=1\left({ }^{2} \mathrm{P}_{3 / 2}\right)$ and $\mathrm{n}=1\left({ }^{2} \mathrm{P}_{1 / 2}\right)$ states $[22]$
Table II Rate Constants and Cross Sections for dissociation of Argon exmiers in gases (adapted from [28])

\begin{tabular}{|c|c|c|}
\hline $\begin{array}{c}\text { Acceptor } \\
(\mathrm{M})\end{array}$ & $\begin{array}{c}\text { Rate Constant } \\
\left(10^{-11} \mathrm{~cm}^{3} / \mathrm{sec}\right)\end{array}$ & $\begin{array}{c}\text { Cross Section } \\
\left(10^{-16} \mathrm{~cm}^{2}\right)\end{array}$ \\
\hline $\mathrm{Kr}$ & 10 & 25 \\
\hline $\mathrm{Xe}$ & 50 & 140 \\
\hline $\mathrm{CH}_{4}$ & 55 & 120 \\
\hline $\mathrm{CO}_{2}$ & 68 & 140 \\
\hline
\end{tabular}

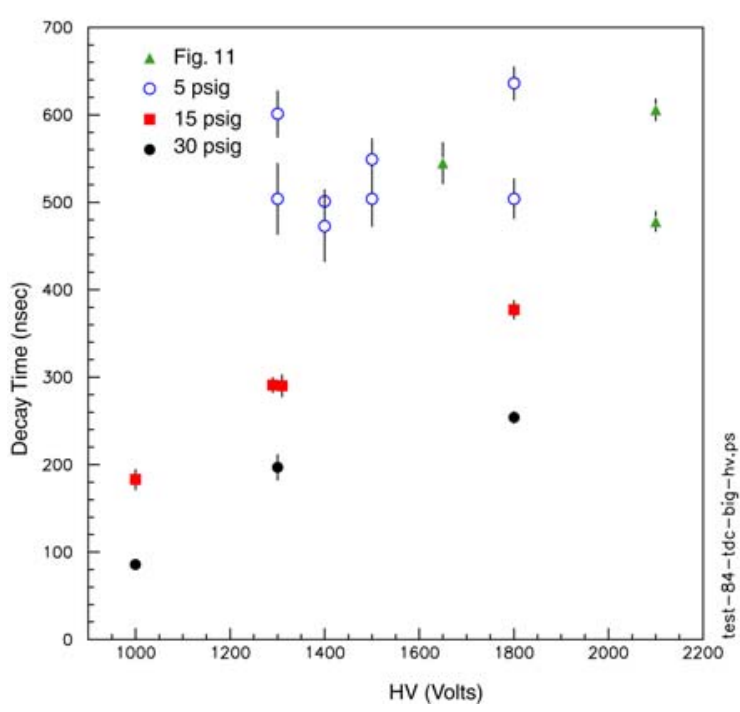

Fig. 15. The variation of the decay time for large pulses is measured as a function of the applied $\mathrm{HV}$ and the pressure. The triangular points are from Fig. 11, also taken at 5 psig with our older DAQ system. The four points at $1800 \mathrm{~V}$ ) have $2.5 \mathrm{ppm} \mathrm{O}_{2}$ added to the nominal $250 \mathrm{ppm}$ $\mathrm{Xe}$ in LAr. The errors are statistical only.

The ionization energies of $\mathrm{Xe}$ and $\mathrm{Kr}$ dissolved in LAr are $10.58 \mathrm{eV}$ and $12.5 \mathrm{eV}$, respectively, and the lowest lying $n=1\left({ }^{2} \mathrm{P}_{3 / 2}\right)$ and $\mathrm{n}=1\left({ }^{2} \mathrm{P}_{1 / 2}\right)$ Ar* states are at $12.0 \mathrm{eV}$ and $12.3 \mathrm{eV}$, respectively [22]. Hence, these $\mathrm{Ar}$ excitons can ionize $\mathrm{Xe}$ but not $\mathrm{Kr}$. Ionization of $\mathrm{Xe}$ or $\mathrm{Kr}$ by collisions with the lowest lying $\mathrm{Ar}_{2}{ }^{*}$ is energetically forbidden [22]. Based on this fact, we expected that more $\mathrm{Kr}$ than $\mathrm{Xe}$ is required to stabilize avalanches in LAr. This was substantiated by our tests, as discussed below.

We observed avalanches in mixtures of LAr and small concentrations of $\mathrm{Kr}$. The signal amplitude depended upon voltage and pressure. With the addition of $\mathrm{Kr}$, stable avalanche signals were observed, but only with higher concentrations of $\mathrm{Kr}$ than of Xe. For smaller concentrations $(<1000 \mathrm{ppm})$ 
LBNL-54763

of $\mathrm{Kr}$, the avalanches were erratic and unpredictable, similar to the behavior with pure LAr.

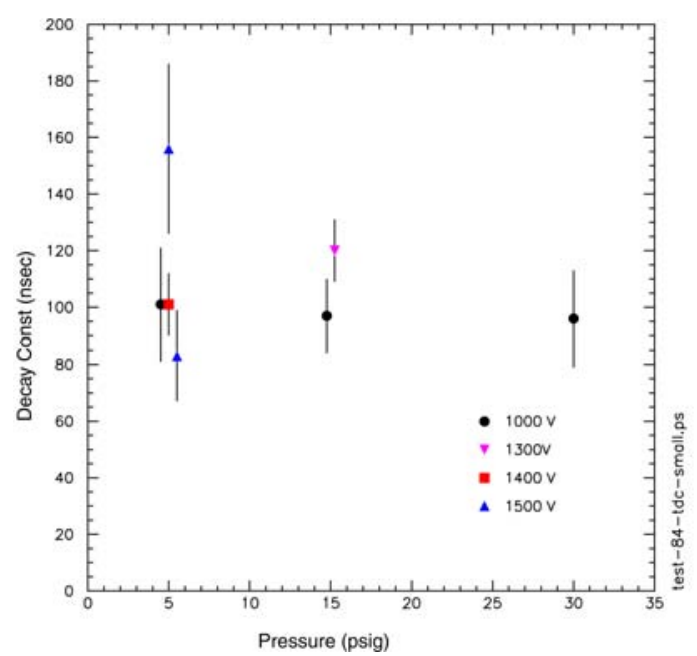

Fig. 16. Decay constants for the small pulses measured as a function of $\mathrm{HV}$ and pressure. The points at 5 psig and 15 psig have been horizontally separated so the error bars are visible. The errors are statistical only, except for the point at $1000 \mathrm{~V}, 15$ psig that has been corrected for a photon fraction of 0.65 and includes the systematic error from the MC correction. The data appear consistent with no dependence of the fit decay constant on either the HV or pressure.

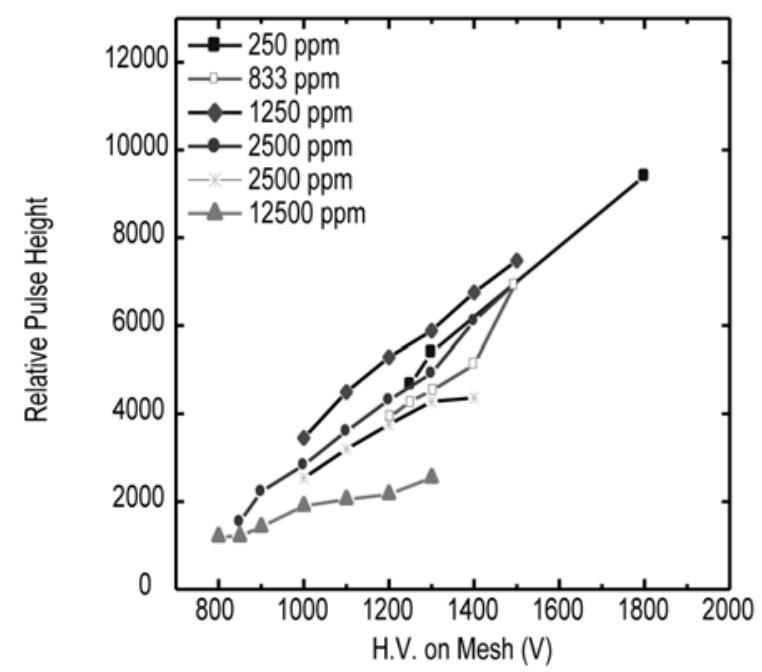

Fig. 17. Pulse height in LAr plus Kr measured versus $\mathrm{HV}$ for various $\mathrm{Kr}$ concentrations, all measured at $5 \mathrm{psig}$.

B. Pulse Amplitude in Krypton Mixtures
The observed pulse heights are approximately linear with HV, as is illustrated in Fig. 17, but with much less dependence on the $\mathrm{Kr}$ concentration than in the case of Xe (Fig. 9). The pulse height in $\mathrm{Kr}$ appears to grow with time (as much as $30 \%$ ). Most of the variation seems to occur in the first $\sim 60$ min after $\mathrm{HV}$ is applied. This effect does not appear to have any apparent dependence on the absolute value of the $\mathrm{HV}$ or $\mathrm{Kr}$ concentration. We have chosen data where the results appeared stable in time.

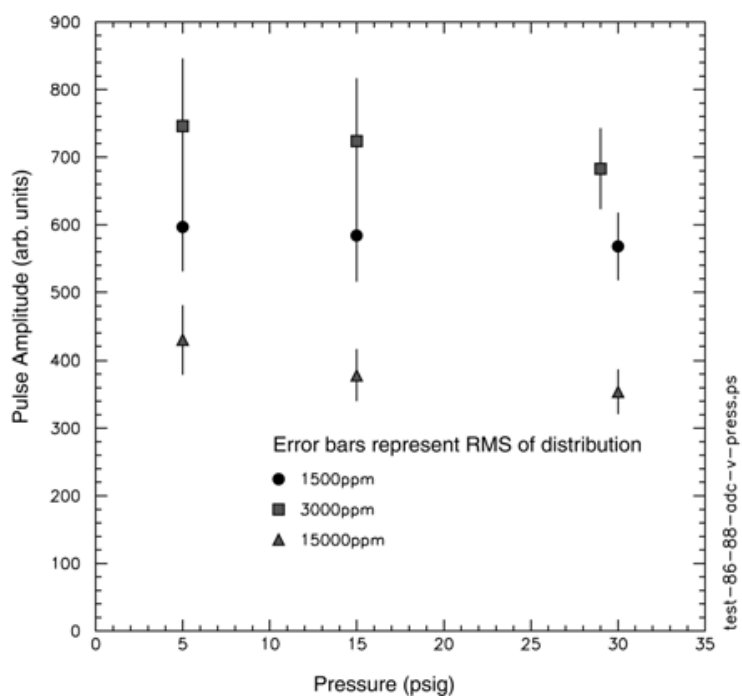

Fig 18. Pressure dependence of signal amplitudes in $\mathrm{LAr} / \mathrm{Kr}$ measured as function of pressure for three separate tests. All of the data was taken were at $1350 \mathrm{~V}$ with the same needle setup. The error bars represent the $r m s$ of the $\mathrm{PH}$ distributions.

With $\mathrm{Kr}$ mixtures, we observed only one set of pulse amplitudes, and these were pressure dependent (Fig. 18). However, the pressure dependence is smaller than for LAr/Xe. The amplitude decreased about $10 \%$ when the gauge pressure was increased from 0 to $1.6 \mathrm{~atm}$. Unlike our experience with $\mathrm{Xe}$, we did not observe pulses that were independent of pressure. There is no monotonic dependence of pulse height on $\mathrm{Kr}$ concentration.

The pulses in Krypton were typically followed by smaller afterpulses, occurring $\sim 10$ to $100 \mu \mathrm{sec}$ after the original pulse (Fig. 19). The rate of afterpulses decreased with decreasing applied voltage. We did not observe this behavior in LAr with Xe, except at the highest voltages near the point of electrical breakdown. 


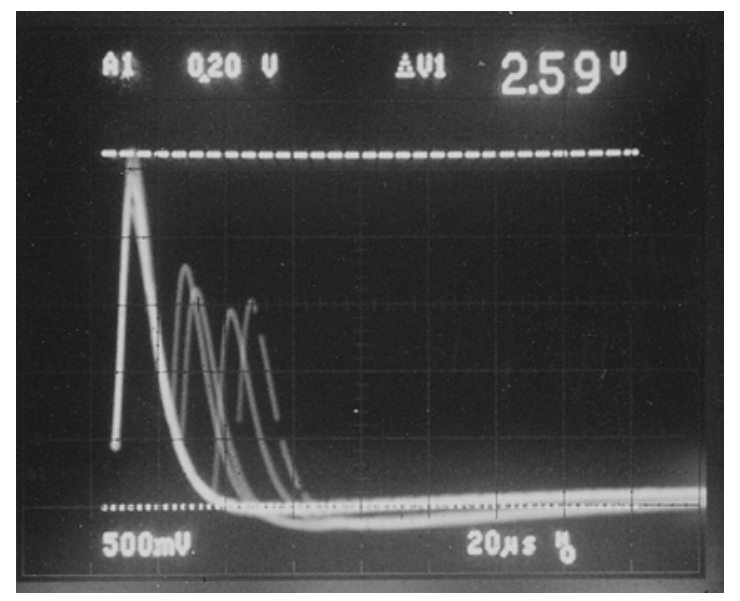

Fig. 19. Photograph of oscilloscope traces of pulses seen in LAr with $2500 \mathrm{ppm} \mathrm{Kr}$ and $1400 \mathrm{~V}$ applied to the drift electrode at $\sim 1.3$ atmospheres pressure (5 psig). First (and largest) pulse is about $2.6 \mathrm{~V}$, and the pulse width of $\sim 10 \mu \mathrm{s}$ fwhm is determined by the amplifier time constants.

\section{Decay Time Spectra vs. Krypton Concentration}

Measurements of decay times for photon emission from avalanches were made for concentrations of 1500, 3000, and 15,000 ppm of $\mathrm{Kr}$ at pressures of 5,15 , and $30 \mathrm{psig}$, using eqn. 7 to fit the data. All these measurements were made at 1350 V. The results are presented in Fig. 20, which shows that there is a decrease in lifetimes as the pressure is increased. Unlike the experience with $\mathrm{Xe} / \mathrm{Ar}$ mixtures, the lifetime values of $\mathrm{LAr} / \mathrm{Kr}$ do not depend strongly on the concentrations used in these tests. These lifetimes are much shorter than those for the Xe/Ar mixtures for the large pulses. The indication of a small but significant pressure dependence of the PH may indicate vapor bubble formation at the needle tip, just as we conjectured for the $\mathrm{Xe} / \mathrm{Ar}$ tests. This hypothesis is one explanation for the decay time dependence on pressure, because the smaller bubbles produced at larger pressure would be expected to disappear more rapidly, leading to shorter ("quenched") decay times.

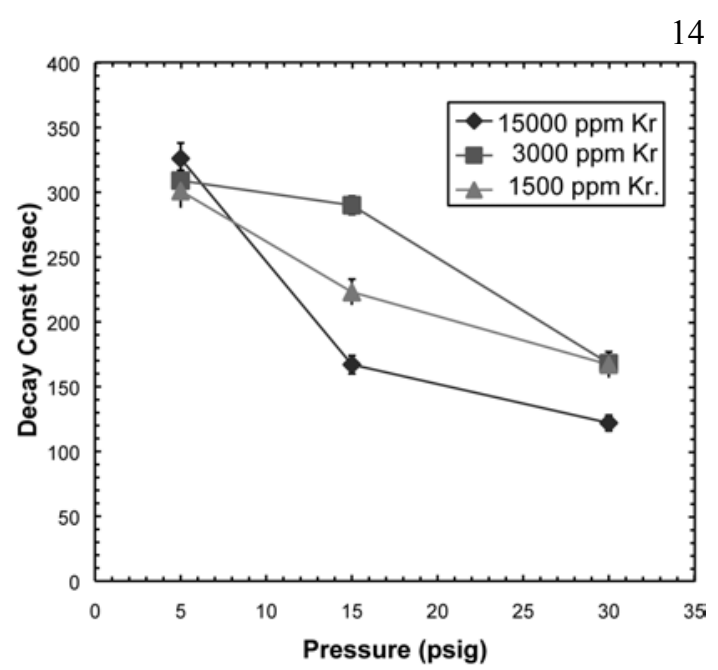

Fig. 20. Decay times for three values of $\mathrm{Kr}$ concentration as a function of pressure, all measured at $1350 \mathrm{~V}$. The errors are statistical only.

\section{Other Measurements}

We tested other additives other than Xe or $\mathrm{Kr}$. Attempts were made to develop a practical detector that would be sensitive over a significant area and not just the narrow region above the tip of the needle in our point-plane geometry.

\section{A. Other Liquid Mixtures}

In the course of our investigations we have made these other measurements discussed here only briefly:

1) We were successful in producing avalanches in LAr containing $\sim 4$ ppm TMS (tetramethylsilane). However, we discontinued testing when we found silicon deposits near the tip of the needle after the test, using electron dispersive $\mathrm{x}$-ray fluorescence (EDXF) surface analysis.

2) Attempts with (30\%) neon in LAr were unsuccessful in producing reliable avalanche behavior.

\section{B. Spindt Cathodes}

We tested an array of $\sim 10,000$ Spindt cathode cells [29], each emitter consisting of a $0.75 \mu \mathrm{m}$ high cone (or "tip") surrounded by a metallic cathode (or "gate") approximately level with the top of the tip (see Fig. 21) and with a circular opening of $0.75 \mu \mathrm{m}$ 


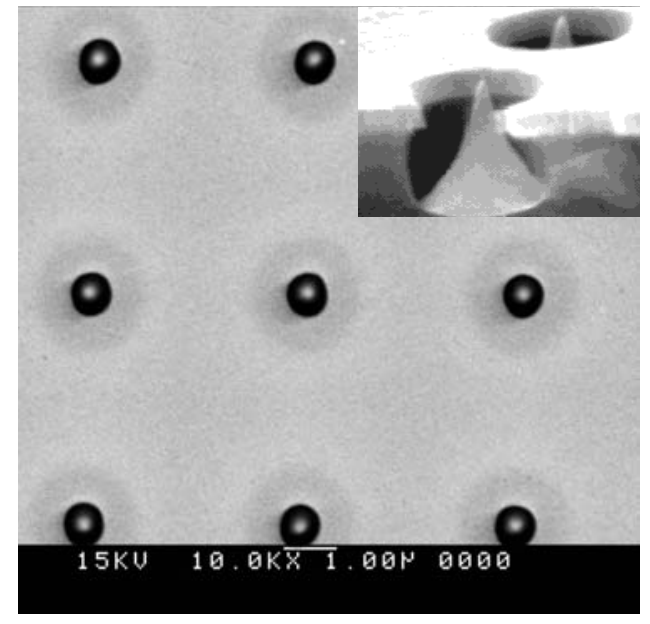

Fig. 21. Micrograph of Spindt cathode surface used in our tests. The hole diameter in the gates is $\sim 0.75 \mu \mathrm{m}$. Inset: cross section through a single cathode showing the metal tip, encircled by a metal gate film and $\mathrm{SiO}_{2}$ insulator fabricated on a silicon substrate (Courtesy, Dr. Capp Spindt, SRI [29]])

diameter. The emitter array has a diameter of $\sim 1$ $\mathrm{mm}$. The Spindt cathodes were placed in the same plane as our ground electrode, but electrically isolated from it. The distance from the ground plane to the drift electrode (cathode) was $6 \mathrm{~mm}$, and the drift field was in the range $1000 \mathrm{~V} / \mathrm{cm}$ to $2000 \mathrm{~V} / \mathrm{cm}$.

Designed for use as a field emission electron source in vacuum, the Spindt cathodes were used here in reverse polarity, so that the cone became an anode to allow electron avalanche multiplication. As a field emission device at $\sim 120 \mathrm{~V}$ tip-gate voltage, electric fields of $\sim 50 \mathrm{MV} / \mathrm{cm}$ have been achieved, about a factor of seven higher than necessary for avalanching in LAr.

We briefly observed $(\sim 20 \mathrm{sec})$ small pulses in $\mathrm{LAr} / \mathrm{Xe}(250 \mathrm{ppm})$ at $\sim 70 \mathrm{~V}$ tip-gate voltage, but these were rapidly followed by breakdown of the device, confirmed by the subsequent large current drawn between the tips and gate at low voltages (5 $12 \mathrm{~V})$. The electrical short between the tips and gate could sometimes be removed by discharging a $1 \mu \mathrm{F}$ capacitor charged to $50 \mathrm{~V}$ across the device. The observed pulses may have been indicators of the incipient breakdown of the device. An alternative explanation is that avalanche multiplication is accompanied by bubble formation at the tip, and the bubble, though very small, creates a conducting path for a spark, resulting in a breakdown or discharge rather than the desired avalanches.

\section{Microstrip Anodes}

We have also tested $0.25 \mu \mathrm{m}$ and $0.40 \mu \mathrm{m}$ wide chromium strip anodes, interdigitated with cathodes 5 $\mu \mathrm{m}$ wide, deposited on a quartz substrate at a pitch of $400 \mu \mathrm{m}$. We saw small pulses $(50$ to $100 \mathrm{mV}$ ) that gradually disappeared over a few minutes. We suspect that the quartz substrate charged up and reduced the applied field. Subsequently, this detector was damaged by a spark discharge and no further tests were done.

\section{Cesium Iodide Photocathode}

We could easily detect photons from the decay of $\mathrm{Xe}^{*}{ }_{2}\left(\mathrm{Ar}_{2}{ }_{2}\right)$ excimers using a gas avalanche detector with a CsI photocathode and a $50 \mu \mathrm{m}$ diameter tungsten wire in $\mathrm{P}-10$ or $\mathrm{CF} 4$ gas. The time resolution was not as good as that of the PMT because of the variation in drift time of the photoelectrons in the gas. For this reason the time measurements reported here were obtained using the VUV PMT.

\section{Discussion and Conclusions}

We have found that small concentrations of Xe, $250 \mathrm{ppm}$ or less, stabilize the electron avalanche process in LAr, which is otherwise erratic with pure LAr. Tests over a wide range of Xe/Ar concentrations characterize the avalanche behavior. The avalanche gains are estimated to be $>100$. We find that there are two modes, one with pressure-dependent signal pulses, and another with smaller, pressureindependent pulses. Our presumption is that the pressure-dependent pulses arise from avalanches occurring in small, transient vapor bubbles produced at the needle tip. The changes in the decay times versus pressure and $\mathrm{HV}$ for these modes tend to support this hypothesis. Both types of pulses have rather narrow pulse height distributions and appear to be saturated, i.e. not proportional to primary ionization. A ${ }^{57} \mathrm{Co}$ source gave a pulse amplitude spectrum very similar to that of a ${ }^{241} \mathrm{Am}$ source, even though the photon energy is about twice as large for ${ }^{57} \mathrm{Co}$ : this is a strong indication of saturation with respect to initial ionization. The pulse height increases approximately linearly with applied voltage above a threshold of $\sim 7 \mathrm{MV} / \mathrm{cm}$. Time spectra of 
photon emission show a pressure and HV dependence not previously seen but cannot be completely reconciled with measurements by others. The photons are sufficiently fast to be used as trigger signals in many applications. Experiments requiring large volumes of Ar will not be limited by the cost of the Xe, because only small concentrations (e.g. 250 ppm) of Xe are needed for stable operation.

We have analyzed the concentration of Xe actually dissolved in the liquid and compared the results with expectations based on the amount of Xe gas added to the LAr. There was good agreement between the expected and analyzed concentrations only when proper mixing and sampling procedures were followed.

Our determination of the positive charge carrier mobility in LAr with small admixtures of Xe is $6.4 \mathrm{x}$ $10^{-3} \mathrm{~cm}^{2} / \mathrm{V}$-sec, in approximate agreement with the mobility measured by others in pure LAr, and we conclude the positive charge carriers are principally holes, not positive ions.

In studying avalanche behavior in LAr with small $\mathrm{Kr}$ admixtures we find only one type of pulse that is pressure dependent. However, the $\mathrm{PH}$ amplitude shows less pressure dependence than with Xe. As in Xe admixtures, we attribute this pressure dependence to avalanches forming in transient gas bubbles. Similar to $\mathrm{Xe}$, the pulse height increases approximately linearly with voltage above the threshold and the decay times depend on the pressure, decreasing as the pressure increases, as would be expected from avalanches in vapor bubbles. The pulse height and time spectra do not depend significantly on the $\mathrm{Kr}$ concentration, which was varied over a wide range. Generally, the concentration of $\mathrm{Kr}$ required for avalanche stabilization is about five times as much as for Xe, and afterpulses are seen much more frequently with $\mathrm{Kr}$.

Neon was also tested as an admixture in LAr, but avalanche signals could not be produced

An attempt to use microstrip anodes of sub-micron dimensions was only partially successful: signals were briefly seen, and then disappeared, presumably from surface charging. Subsequently, sparking damaged this detector

We were unsuccessful in producing reliable avalanches using a Spindt cathode device operated in reverse polarity. A design that we believe might be successful would scale up the cell size by a factor of $\sim 10-100$, so that small bubbles $(\leq 1 \mu \mathrm{m})$, if present, do not lead to electrical discharges between the tips and gate. Structures of this size would be able to provide position resolution of order 10-30 $\mu \mathrm{m}$, and would have capability of a practical detector.

\section{Acknowledgments}

We thank S. Derenzo for several informative discussions, and J. Wise for clarifying Xe solubility in Ar. Also, we thank E. Saiz for help with the electron microscope facility, and professors K. Abe and H. Yoshimoto for supplying samples of TMS and TMG. Dr. Keith H. Jackson of the LBNL Materials Science Division manufactured the micro strip detectors for us.

\section{REFERENCES}

[1] C. Brassard, "Liquid ionization detectors," Nucl. Instr. Meth., vol. 162, pp. 29-47, 1979.

[2] P. Cennini, S. Cittolin, J-P. Revol, C. Rubbia, W-H. Tian, P. Picchi, et al., "Performance of a three-ton liquid argon time projection chamber," Nucl. Instr. Meth., vol. A345, pp. 230243, 1994.

[3] S. E. Derenzo, T. S. Mast, H. Zaklad, R. A. Muller " Electron avalanche in liquid xenon," Phys. Rev. A, vol. 9, pp.25822591, 1974 and, for example, A..P.L. Policarpo, V. Chepel, M.I. Lopes, V. Peskov, P. Geltenbort, R. Ferreira Marques, et.al., "Observation of electron multiplication in liquid xenon with microstrip plate,"Nucl. Instr Meth., vol. A365, pp. 568571,1995 .

[4] ${ }^{57} \mathrm{Co}$ and ${ }^{241} \mathrm{Am}$ Source purchased from Isotope Products, Inc., Burbank, CA 91504, USA.

[5] EMR Schlumberger vacuum UV PMT Model 541F-09-17, 20 Wallace Road, Princeton Jct., NJ 08550, USA.

[6] Matheson 4A molecular sieve (model 461), Matheson TriGas, 166 Keystone Drive, Montgomeryville, PA 18936. In addition to $2.6 \mathrm{ppm} \mathrm{O}_{2}$, the gas analysis supplied by the manufacturer lists the total hydrocarbon concentration less than $0.1 \mathrm{ppm}$ (not detectable), Freon 11 less than $0.1 \mathrm{ppm}$ (not detectable) and less than $1.0 \mathrm{ppm}_{2} \mathrm{O}$.

[7] Messer Oxysorb $\mathrm{O}_{2}$ filter Model SS11-HV, 5275 Tighman St., Allentown, PA 18104, USA.

[8] Gatekeeper inert gas purifier, Aeronex Inc, 6975 Flanders Dr, San Diego, CA 92121, USA.

[9] Model 316R Trace Oxygen Analyzer, Teledyne Analytic Instruments, 16830 Chestnut St., City of Industry, CA 917491580, calibrated with a certified mixture of Argon with $0.520 \mathrm{ppm} \mathrm{O}_{2}$ obtained from Bay Airgas, 1588 Dolittle Dr., San Leandro, CA. The lowest sensitivity of the $\mathrm{O}_{2}$ meter is $10 \mathrm{ppb}$.

[10] Analysis performed by Atlantic Analytical Laboratory, P.O. Box 220, Salem Industrial Park, Building \#4, Whitehouse, NJ 08888, USA.

[11] W. R. Smythe, Static and Dynamic Electricity, Section 5.28, McGraw-Hill, NY, NY, 1939.

[12] Picoammeter (model 485), Keithley Instruments Inc, 28775 Aurora Ave., Cleveland, OH 44139, USA.

[13] LabView program version 2.2.1, National Instruments, 6504 Bridge Point Pkwy, Austin, TX 78730, USA.

[14] PCA3 version 2.31, Oxford Instrument, 601 Oak Ridge Turnpike, Oak Ridge, TN 37831, USA. 
[15] eV Products, Model 550, type 5093 Division of II-IV Incorporated, 373 Saxonburg Blvd., Saxonburg, PA 16056, USA.

[16] K. Arii and W. Schmidt, "Current Injection and Light Emission in Liquid Argon and Xenon in a Divergent Electric Field”, IEEE Trans. on Elec. Insul., vol. EI-19, no.1, pp. 1623, Feb. 1984.

[17] MAXWELL Electric Field Simulator, Ansoft Corporation, Four Station Square Suite 200, Pittsburgh, PA 15219, USA.

[18] B. Halpern and R. Gomer, "Field Emission in Liquids", J. of Chem. Phys., vol. 51, no.3, pp. 1031-1047, Aug. 1969.

[19] C. R. Gruhn and R. Loveman, "A review of the physical properties of liquid ionization chamber media," IEEE Trans. Nucl. Sci., vol. NS-26, pp. 110-119, 1979.

[20] Hilt and W. Schmidt, "Positive hole mobility in Liquid Xenon", Chem. Phys., vol. 183, pp. 147-153, May. 1994.

[21] S. E. Derenzo, D. B Smith, R. G. Smits, H. Zaklad, L. A. Alverez, "Recent Developments in High Resolution Counters," Lawrence Berkeley National Laboratory (LRL Preprint), UCRL-20118, September, 1970 and Fermi National Accelerator Laboratory (NAL Preprint) Summer Study Report SS181, July 1970.

[22] S. Kubota, A. Nakamoto, T. Takahashi, S. Konni, T. Hamada, M. Miyajima, et al, "Evidence of the existence of exciton states in liquid argon and exciton-enhanced ionization from xenon doping", Phys. Rev. B, vol. 13, no.4, pp. 1649-1653, Feb. 1976.

[23] M. Miyajima, T. Takahashi, S. Konno, T. Hamada, S. Kubota, H. Shibamura, T. Doke., "Average energy expended per ion pair in liquid argon," Phys. Rev. A, vol. 9, pp.14381443, 1974.

[24] S. Kubota, M. Hishida, M. Suzuki, J-z. Ruan(Gen), "Liquid and solid argon, krypton and xenon scintillators," Nucl. Instr. Meth. Vol. 196, pp. 101-105, 1982.

[25] E. Conti, G. Carugno, and A. Intravaia, "Time behaviour of the scintillation light in mixtures of liquid argon and xenon," Nucl. Instr. Meth., vol. A382, pp. 475-478, 1996.

[26] E. Conti, private communication.

[27] see for example: S. Kubota, A Nakamoto, T. Takahashi, T. Hamada, E. Shibamura, M. Miyajima, K. Masuda and T. Doke, " Recombintation Luminescence in liquid argon and liquid xenon," Phys Rev. B, Vol 17, pp. 2762-2765, June 1977, and S. Kubota, M. Hishida, J-z. Raun(Gen), "Evidence for a triplet state of self-trapped exciton states in liquid argon, krypton and xenon", J. Physics C: Solid State Phys., vol. 11, pp 2545-2651, Feb 1978.

[28] T. Oka, M. Kogoma, M. Imamura, and S. Arai, "Energy transfer of argon excited diatomic molecules", J. Chem. Phys, vol. 70, no.7, pp. 3384-3389, Apr. 1979.

[29] Provided by, and the intellectual property of SRI, contact Dr. Capp Spindt, director of Vacuum Microelectronics Program, Applied Physics Sciences Laboratory, SRI, 333 Ravenswood Ave, Menlo Park, CA 94025, USA. 